\title{
Dynamics of soliton solutions of the fifth-order nonlinear Schrödinger equation via the Riemann-Hilbert approach
}

\author{
Jin-Jie Yang ${ }^{1}$, Shou-Fu Tian ${ }^{1}$, TIan-Tian Zhang ${ }^{1}$, and Xiao-Li Wang ${ }^{2}$ \\ ${ }^{1}$ China University of Mining and Technology \\ ${ }^{2}$ Qilu University of Technology
}

January 24, 2021

\begin{abstract}
The theory of inverse scattering is developed to investigate the initial-value problem for the fifth-order nonlinear Schrödinger (foNLS) equation under the zero boundary conditions at infinity. The spectral analysis is performed in the direct scattering process, including the establishment of the analytical, asymptotic and symmetric properties of the scattering matrix and the Jost functions. In the inverse scattering process, a suitable Riemann-Hilbert (RH) problem is successfully established by using the modified eigenfunctions and scattering data, and the relationship between the potential function and the solution of the $\mathrm{RH}$ problem is successfully established. In order to further analyze the propagation behavior of the solutions of the foNLS equation, we present some new phenomena of studying the one-, two-, and three- soliton solutions corresponding to simple zeros in scattered data. Finally, we also analyze the one- and two-soliton solutions corresponding to double zeros.
\end{abstract}

\section{Hosted file}

2020-foNLS-MMAS.pdf available at https://authorea.com/users/302440/articles/505561-dynamicsof-soliton-solutions-of-the-fifth-order-nonlinear-schr\%C3\%B6dinger-equation-via-theriemann-hilbert-approach 